\title{
Application Article \\ Bandwidth Improvement of EBG Resonator Antennas Using Double-Layer FSS
}

\author{
Lina Moustafa and Bernard Jecko \\ XLim Laboratory, OSA Department, University of Limoges, 123 Avenue Albert Tomas, 87060 Limoges Cedex, France \\ Correspondence should be addressed to Lina Moustafa, lina.moustafa@xlim.fr
}

Received 10 June 2008; Revised 27 September 2008; Accepted 12 November 2008

Recommended by Lance Griffiths

A double-layer frequency selective surface (FSS) is proposed as a means to enhance the bandwidth of an electromagnetic band gap (EBG) resonator antenna. Due to its inverted reflection phase variation and its wide selectivity bandwidth, the structure used in the radiating wall of the resonator allows increasing the radiating bandwidth of the last one. The resonator is fed by a patch feeding source placed inside the cavity at the proximity of its metallic ground. The antenna bandwidth is significantly improved by virtue of employing the double-layer FSS. Modelled results of an antenna working at $5 \mathrm{GHz}$ are shown.

Copyright (C) 2008 L. Moustafa and B. Jecko. This is an open access article distributed under the Creative Commons Attribution License, which permits unrestricted use, distribution, and reproduction in any medium, provided the original work is properly cited.

\section{INTRODUCTION}

In the past few years, new solutions that combine directivity and simple feeding mechanism have been proposed to design compact antennas. Dielectric or metallic arrays have been placed over a perfect electric ground. EBG resonator antennas have been formed [1-4]. While important gain values have been realised, the antenna profile has been kept compact (close to the half wavelength). One drawback of this system is its narrow bandwidth; only one frequency and a limited range around this central frequency satisfy the resonant model. Recent works presented by Feresidis and Vardaxoglou [5] have demonstrated a bandwidth enhancement of a resonator antenna, in which the antenna thickness was equal to the operating wavelength. They used combined FSS to create the cavity radiating wall. In this work, we propose improving the bandwidth of a $\lambda / 2$ EBG resonator antenna using double-layer FSS as the cavity radiating wall. The design of used frequency selective surfaces is first discussed. Second, the optimised resonant structure that allows improving bandwidth of printed microwave antennas is described.

\section{EBG RESONATOR PRINCIPLE}

EBG resonator antennas are good candidates to answer compactness and high-directivity requirements of many applications. These antennas are easy to be fabricated since they use dielectric or metallic slabs periodically arranged over a grounded feeding source. Their compactness can be even more improved if the cavity walls design includes frequency selective surfaces [6]. Whether the radiating wall material is dielectric or metallic, a simple optical ray model can be used to depict the cavity modes in the normal direction. If we consider multiple reflections of the wave emitted by the feed, a resonance is achieved when rays projected through the radiating aperture have equal phases. The equation determining the resonance condition for a maximum of directivity in the normal direction is

$$
\varphi_{\text {up }}+\varphi_{\text {low }}-2 k h=2 n \pi, \quad n=0,1,2, \ldots
$$

$\varphi_{\text {up }}$ is the reflection phase of the resonant cavity upper wall. $\varphi_{\text {low }}$ is the reflection phase of the resonator lower wall. In general, EBG resonator design has metallic ground as the lower wall, and $\varphi_{\text {low }}$ is then equal to $\pi$. $h$ is the resonant height of the cavity; $k$ is the propagation constant. This equation underlines the presence of a resonance in the $z$ direction.

If the wavelength is fixed, the height is determined in function of $\varphi_{\text {up }}$. Usually, the phase decreases linearly with frequency, while $2 k h$ increases and in consequence (1) is satisfied for only one frequency and a narrow band around this frequency. 


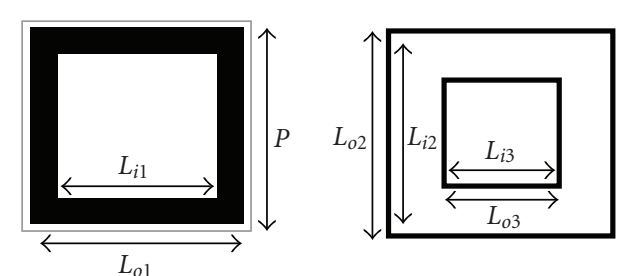

FiguRE 1: FSS1 and FSS2 unit cells geometry.

If (1) is verified for a wide frequency band, the general behaviour of the structure should be also wideband. To answer the wideband requirement, the phase of the resonator upper wall should be the following $\varphi_{\text {up }}=2 k h(n=0)$.

For a given value of $h$, the operating bandwidth can be enlarged by minimising $\varphi_{\text {up }}(f)-2 k h$, this can be realised by reducing the $\varphi_{\text {up }}(f)$ curve slope in a frequency range as wide as possible and by inverting the phase variation versus frequency. The linear increase of $\varphi_{\text {up }}(f)$ is more advantageous in terms of resonator bandwidth. While $2 \mathrm{kh}$ increases with frequency, $\varphi_{\text {up }}(f)$ increases too, and (1) can be satisfied in a very wide frequency band.

\section{CAVITY WALLS CHOICE}

Based on the above analysis, the lower wall of the resonator is left unchanged while its upper wall is replaced by a double-layer FSS. It has been shown that combining FSS offers original reflection properties and particularly allows inverting the phase slope [5].

\subsection{Double-layer FSS design}

The optimised resonator upper wall is composed of two layers of FSS separated by a distance of $d$. Low layer FSS1 is a stop band FSS with square loop elements. The unit cell has outer length $L_{o 1}=26 \mathrm{~mm}$ and inner length $L_{i 1}=19 \mathrm{~mm}$. They are periodically arranged in $x$ - and $y$-directions with a periodicity of $P=28 \mathrm{~mm}$. A study of the FSS reflectivity has been done, and reflection coefficient magnitude and phase are shown in Figures 2 and 3. As illustrated in Figure 2, the reflection coefficient magnitude $|R 1|$ varies between a value of 0.85 and 1 in the operating frequency band around $5 \mathrm{GHz}$ $(4.5-5.5 \mathrm{GHz})$, and the reflection phase $\varphi_{1}$ of FSS1 is positive $\left(150^{\circ} \sim 170^{\circ}\right)$ and it decreases with frequency (see Figure 3 ).

High layer FSS2 is a pass band FSS made of a metallic grid fitted out with square loops. The grid unit cells have outer length $L_{02}=28 \mathrm{~mm}$ and inner length $L_{i 2}=26 \mathrm{~mm}$ (see Figure 1). Square loops inside the grid have $L_{03}=15 \mathrm{~mm}$ and $L_{i 3}=13 \mathrm{~mm}$. Reflectivity of the FSS is studied; the reflection coefficient magnitude $|R 2|$ varies between a value of 0 and 0.55 in the frequency band of interest $(4.5 \mathrm{GHz}-5.5 \mathrm{GHz})$ (see Figure 2), and the reflection phase $\varphi_{2}$ of the surface is negative $\left(-125^{\circ} \sim-90^{\circ}\right)$ and it decreases with frequency (see Figure 3).

Association of FSS1 and FSS2 can produce inverted reflection phase slope, if the reflection magnitudes of FSS layers have opposite variation with frequency. In addition,

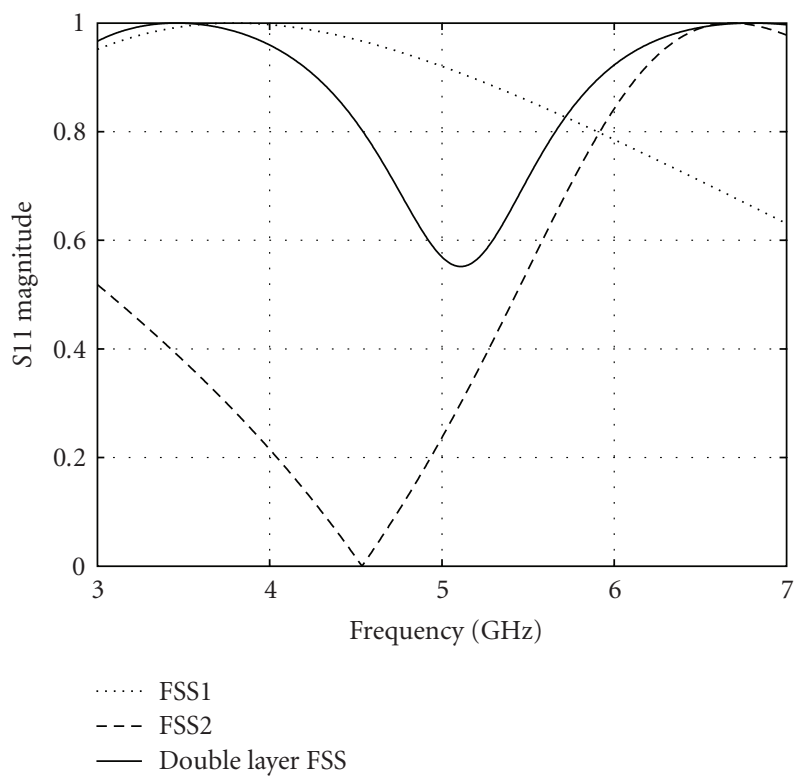

FIGURE 2: Reflection coefficient magnitude.

to provide this particular phase shape, FSS1 and FSS2 have to be separated by a distance of $d$ calculated using (1) with $\left(\varphi_{\text {low }}=\varphi_{1}, \varphi_{\text {up }}=\varphi_{2}\right)$; this behaviour is the result of a resonance installed between the FSS layers:

$$
\phi_{1}+\phi_{2}-2 k d=0
$$

$d$ is thus equal to $4 \mathrm{~mm}$ at the operating frequency. $\varphi_{1}$ and $\varphi_{2}$ have to be almost equal and of opposite sign to achieve a value of $d$ as small as possible.

Reflectivity of the double-layer FSS has been analysed. The examination of the double-layer FSS reflection coefficient is necessary to determine the appropriate height between the last one and the metallic ground to form the resonator.

As shown in Figures 2 and 3, the behaviour in terms of reflection of the double-layer FSS is remarkable; it has the minimum value of magnitude and an increasing phase in the frequency band around $5 \mathrm{GHz}$. The reflection coefficient magnitude varies between 0.55 and 1 in the analysis band; it is equal to 0.55 at $5 \mathrm{GHz}$ and increases by moving away from this frequency. The resonator formed between the studied material and the ground plane is more selective and less wideband when the reflection coefficient tends to 1 . The reflection phase has a particular shape in the frequency range $4.8-5.4 \mathrm{GHz}$. In this band, the phase rises from $80^{\circ}$ to $100^{\circ}$ and it is equivalent to a phase excursion of $20^{\circ}$ in a band of $12 \%$.

Consequently, to obtain increasing reflection phase, double frequency selective surface layers are needed and they should be combined with appropriate separating distance calculated in a manner to create a resonance between layers. The low layer determines in part the reflectivity of the final structure (its reflection coefficient magnitude) and a resonant layer with low reflectivity, and a negative phase is needed to realise compact resonator upper wall. 


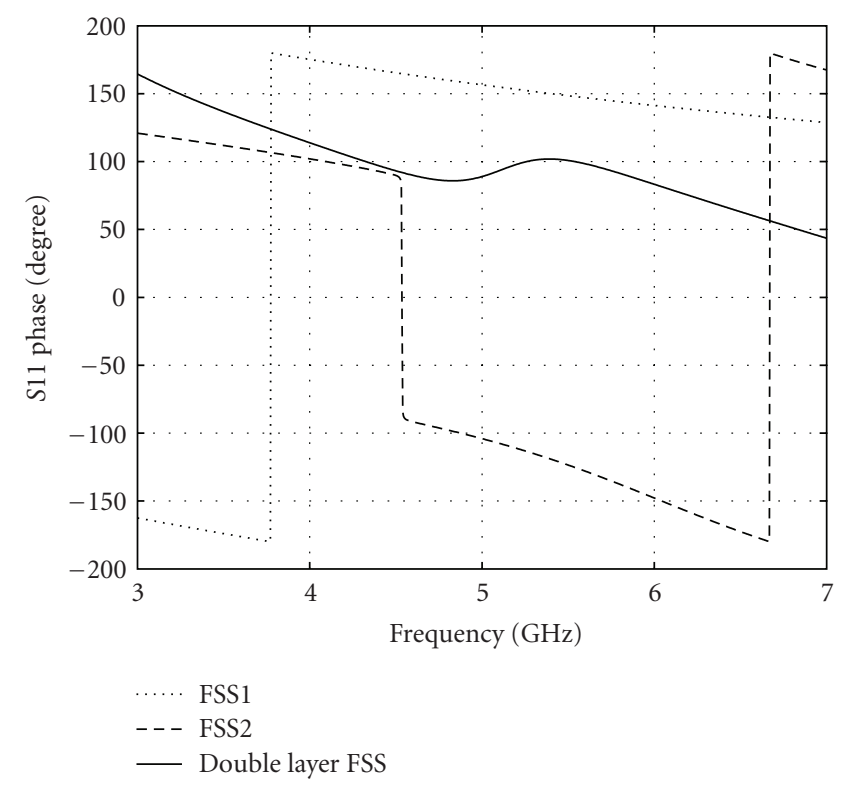

Figure 3: Reflection coefficient phase.

The resonance of the high layer is chosen to be lower or higher than the operating frequency but not at this frequency. The high layer can then be totally transparent.

Geometry of the upper wall unit cell is chosen in a manner to have reasonable antenna directivity value (around $15 \mathrm{dBi}$ ) at $5 \mathrm{GHz}$. This value is the function of a doublelayer FSS reflection coefficient magnitude and thus of each layer reflection magnitude. A directivity value of $15 \mathrm{dBi}$ corresponds to a 0.55 total reflection magnitude value.

\subsection{Resonator transfer function}

The material studied above can be now used to realise an EBG resonator. The double-layer FSS is employed as the resonator upper wall. The resonant height is calculated using (1) (see Figure 4) with $\varphi_{\text {up }}$ (the reflection phase of the double-layer FSS), $\varphi_{\text {low }}$ (the reflection phase of the metallic ground), and $h$ (the resonant height). As we can notice, there is a frequency range for which $h$ is almost constant ( $h=27 \mathrm{~mm}$ ). The resonant height is maintained constant in a frequency range of about $20 \%$ around $5 \mathrm{GHz}$. Equation (1) is satisfied in a wide band of frequencies.

Once the height is determined, it is possible to predict the operating bandwidth of the antenna. An approximate value can be found by solving (3). It can be calculated at $-3 \mathrm{~dB}$ of maximum value of selectivity.

The function $S(f)$ characterises the frequency response of the EBG structure. Its calculation allows the estimation of the resonator intrinsic operating bandwidth without taking into consideration the quality of the feeding source. $S(f)$ can be calculated by considering the sum of the contributions outgoing from the upper wall while exciting the resonator by a plane wave source. This takes in consideration the multiple paths of the electromagnetic waves between the EBG material

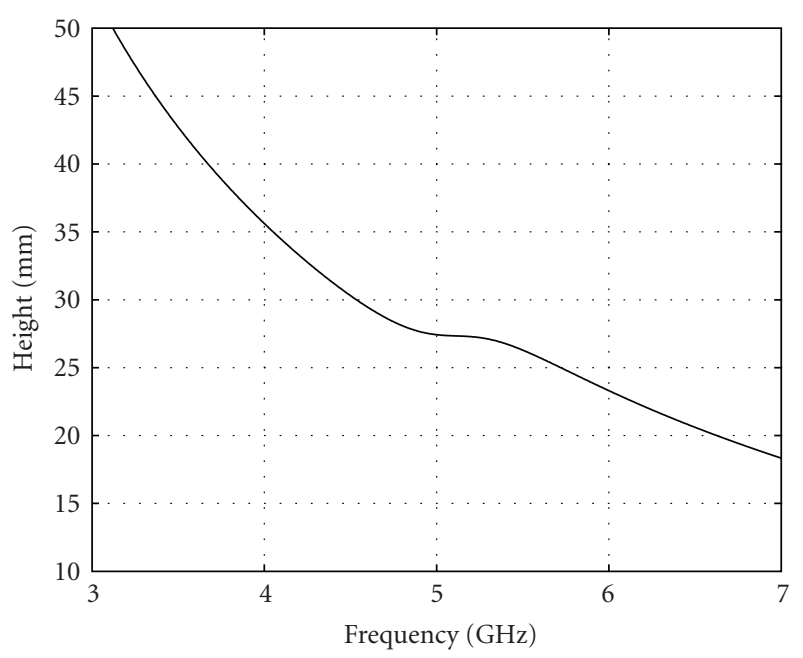

FIGURE 4: Height of the EBG resonator.

and the metallic plane. By using Trentini model, for fixed polarisation and normal incidence, we get

$$
S(f)=\frac{T(f) \exp \left(-j k_{0} h\right)}{1+R(f) \exp \left(-j 2 k_{0} h\right)} .
$$

$T(f)$ and $R(f)$ are, respectively, the transmission and reflection coefficients of the double-layer FSS, $k_{0}$ is the wave vector, $h$ is resonator height.

As we can see in Figure 5, the formed resonator has a pass- band filtering function in a wide range of frequencies limited by $f_{\text {low }}=4.6 \mathrm{GHz}$ and $f_{\text {up }}=5.7 \mathrm{GHz}$ and equivalent to $22 \%$ of band. It is the resonator bandwidth that can likely be obtained using the studied double-layer FSS. The shape of the resonator antenna directivity is not the same as for its selectivity because the source contribution is not determined in (3).

\section{ANTENNA PERFORMANCE}

The optimised FSSs separated by a distance of $d=4 \mathrm{~mm}$ are used to design the antenna upper wall. They are placed at a distance of $h=27 \mathrm{~mm}$ from a perfect mirror. Formed resonator is fed by a single patch source (see Figure 6). Lateral size of the antenna is $21 * 21 \mathrm{~cm}^{2}$ (about $3.5 \lambda * 3.5 \lambda$ at $5 \mathrm{GHz}$ ).

Simulations of the whole antenna have been carried out using the FDTD method. The directivity of the prototype is shown in Figure 7. A maximum directivity value of about $15 \mathrm{dBi}$ has been obtained at $5.5 \mathrm{GHz}$, the directivity bandwidth at $-3 \mathrm{~dB}$ of maximum directivity is equal to $1.3 \mathrm{GHz}$ (about $25 \%$ from $4.2 \mathrm{GHz}$ to $5.5 \mathrm{GHz}$ ). The directivity rises quickly from $12 \mathrm{~dB}$ at $4.2 \mathrm{GHz}$ to attend $15 \mathrm{~dB}$ at the cut frequency $5.5 \mathrm{GHz}$, frequency after which the axial directivity decreases and the radiation patterns deflect. A bandwidth enhancement of about $12 \%$ in comparison to classical EBG antenna designs, with same value of directivity, has been obtained.

Radiation patterns in the E- and H-plane are shown at the centre- and near-edges frequencies of the operating 


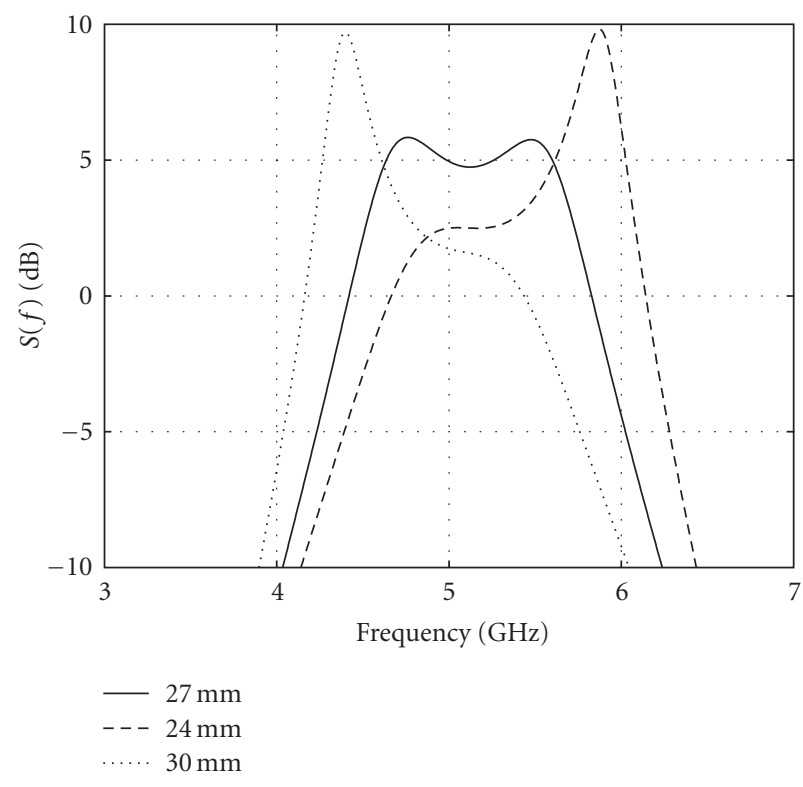

FIGURE 5: Selectivity of the resonator for normal incidence and various resonant height values.

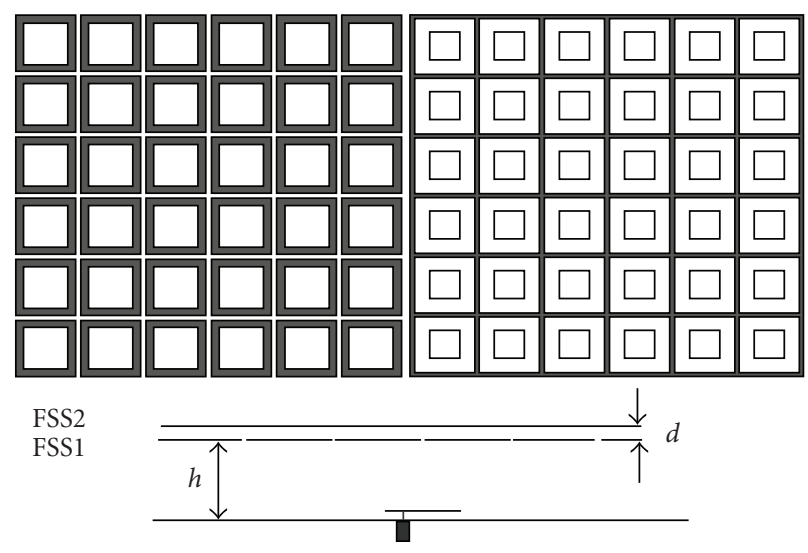

FIGURE 6: Schematic design of the FSS layers and the resonator antenna formed between the double-layer FSS and the ground.

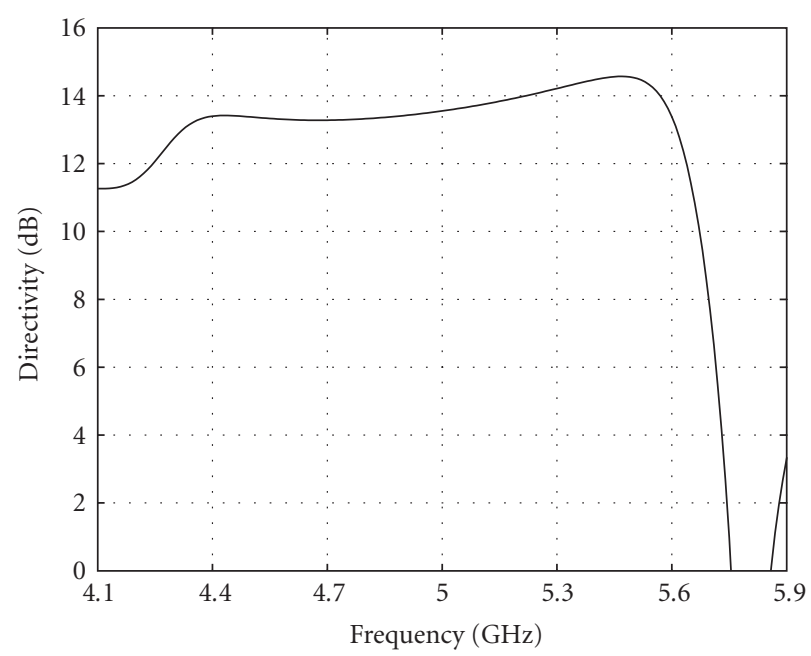

FIGURE 7: Directivity versus frequency.

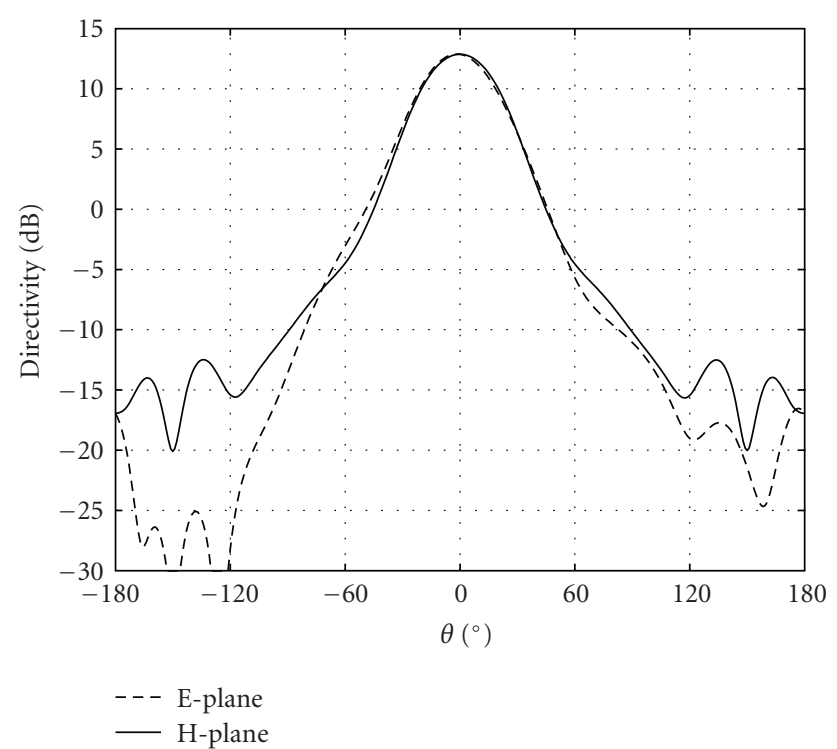

Figure 8: Radiation patterns at the lower end frequency $(4.5 \mathrm{GHz})$ of the operating bandwidth in the E- and H-plane.

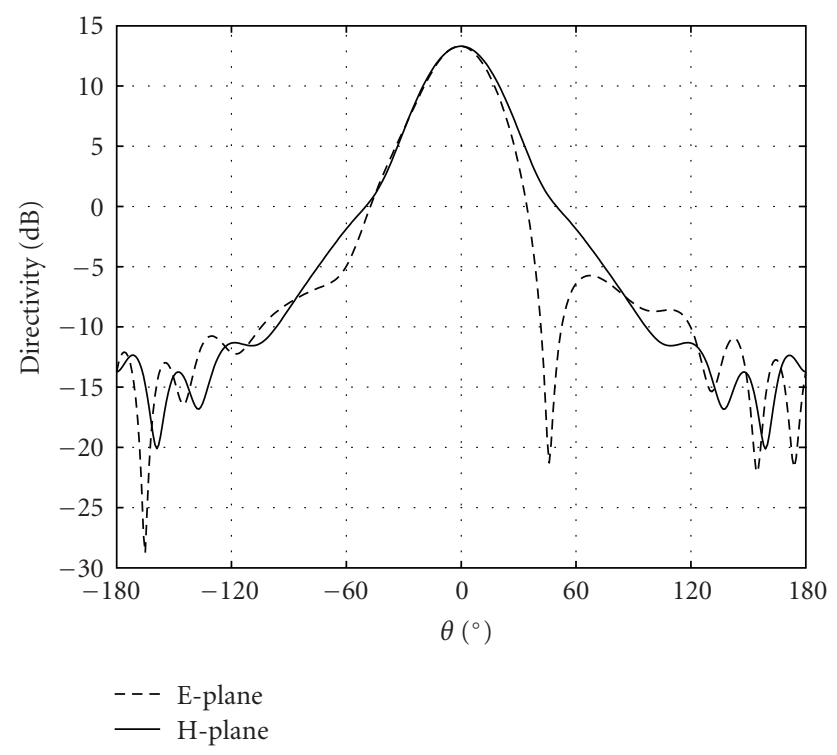

FIGURE 9: Radiation patterns at the central frequency ( $5 \mathrm{GHz}$ ) of the operating bandwidth in the E- and H-plane.

bandwidth (see Figures 8, 9, and 10). Side lobe levels are below $-20 \mathrm{~dB}$ in both planes, they only rise to $-15 \mathrm{~dB}$ at the upper end frequencies of the band and particularly in the $\mathrm{H}$ plane.

We can underline that the directivity bandwidth is in accordance with the bandwidth determined using the resonator selectivity function $(22 \%)$ even the shape is not exactly the same. The directivity takes into account the effect of the source. It explains the difference with the frequential selectivity that only predicts the frequential response of the resonator 


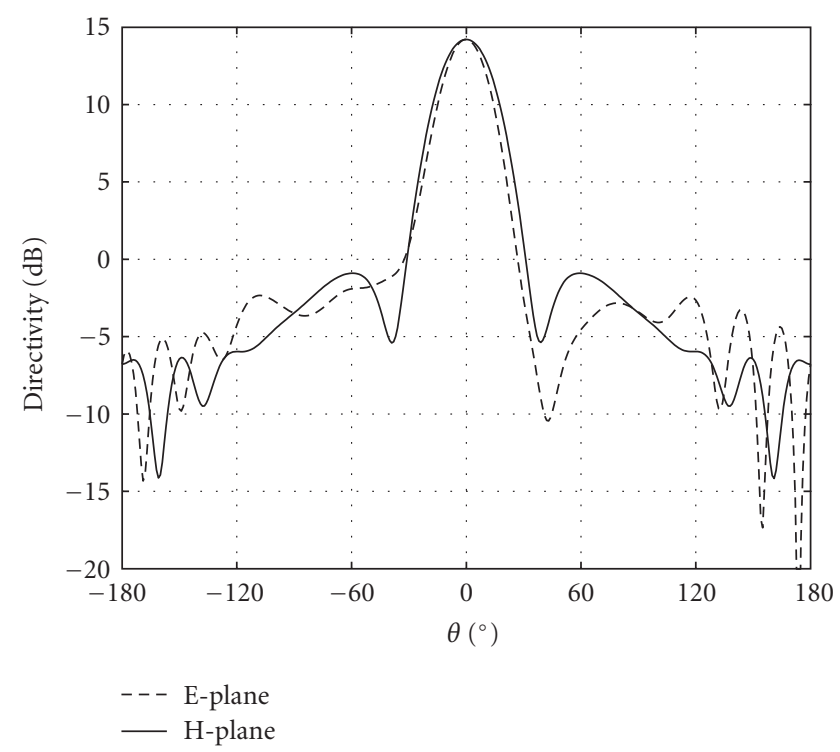

FIGURE 10: Radiation patterns at the upper end frequency $(5.5 \mathrm{GHz})$ of the operating bandwidth in the E- and H-plane.

\section{CONCLUSION}

An optimised design of a broadband EBG resonator antenna using double-layer FSS has been presented. The double-layer FSS has replaced the classical upper wall of the resonator design. Combining FSS with particular separating distance has allowed inverting the reflection phase variation with frequency, thus enlarging the selectivity of built resonator. Formed EBG resonator antenna has wider bandwidth than classical EBG resonator antenna design. A $12 \%$ of bandwidth enhancement has been realised.

\section{REFERENCES}

[1] G. V. Trentini, "Partially reflecting sheet arrays," IRE Transactions on Antennas and Propagation, vol. 4, no. 4, pp. 666-671, 1956.

[2] J. R. James, S. J. A. Kinany, P. D. Peel, and G. Andrasic, "Leakywave multiple dichroic beamformers," Electronics Letters, vol. 25, no. 18, pp. 1209-1211, 1989.

[3] C. Cheype, C. Serier, M. Thevenot, T. Monediere, A. Reineix, and B. Jecko, "An electromagnetic band gap resonator antenna," IEEE Transactions on Antennas and Propagation, vol. 50, no. 9, pp. 1285-1290, 2002.

[4] L. Leger, C. Serier, R. Chantalat, M. Thevenot, T. Monediere, and B. Jecko, "1D dielectric EBG resonator antenna design," Annales des Télécommunications, vol. 59, no. 3-4, pp. 242-260, 2004.

[5] A. P. Feresidis and J. C. Vardaxoglou, "A broadband high-gain resonant cavity antenna with single feed," in Proceedings of the 1st European Conference on Antennas and Propagation (EuCAP '06), pp. 1-5, Nice, France, November 2006.

[6] A. Ourir, A. de Lustrac, and J.-M. Lourtioz, "Optimization of metamaterial based subwavelength cavities for ultracompact directive antennas," Microwave and Optical Technology Letters, vol. 48, no. 12, pp. 2573-2577, 2006. 

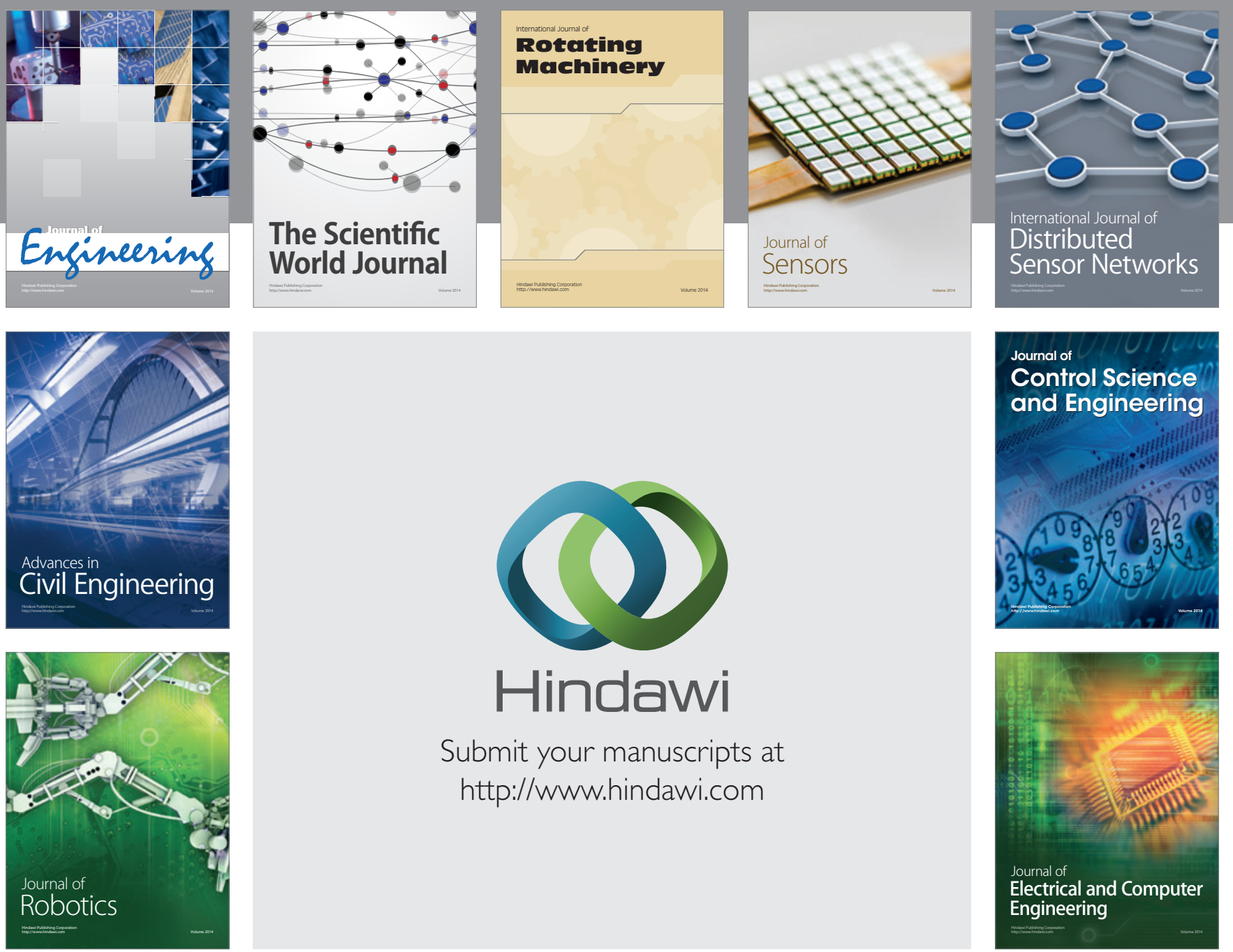

Submit your manuscripts at

http://www.hindawi.com
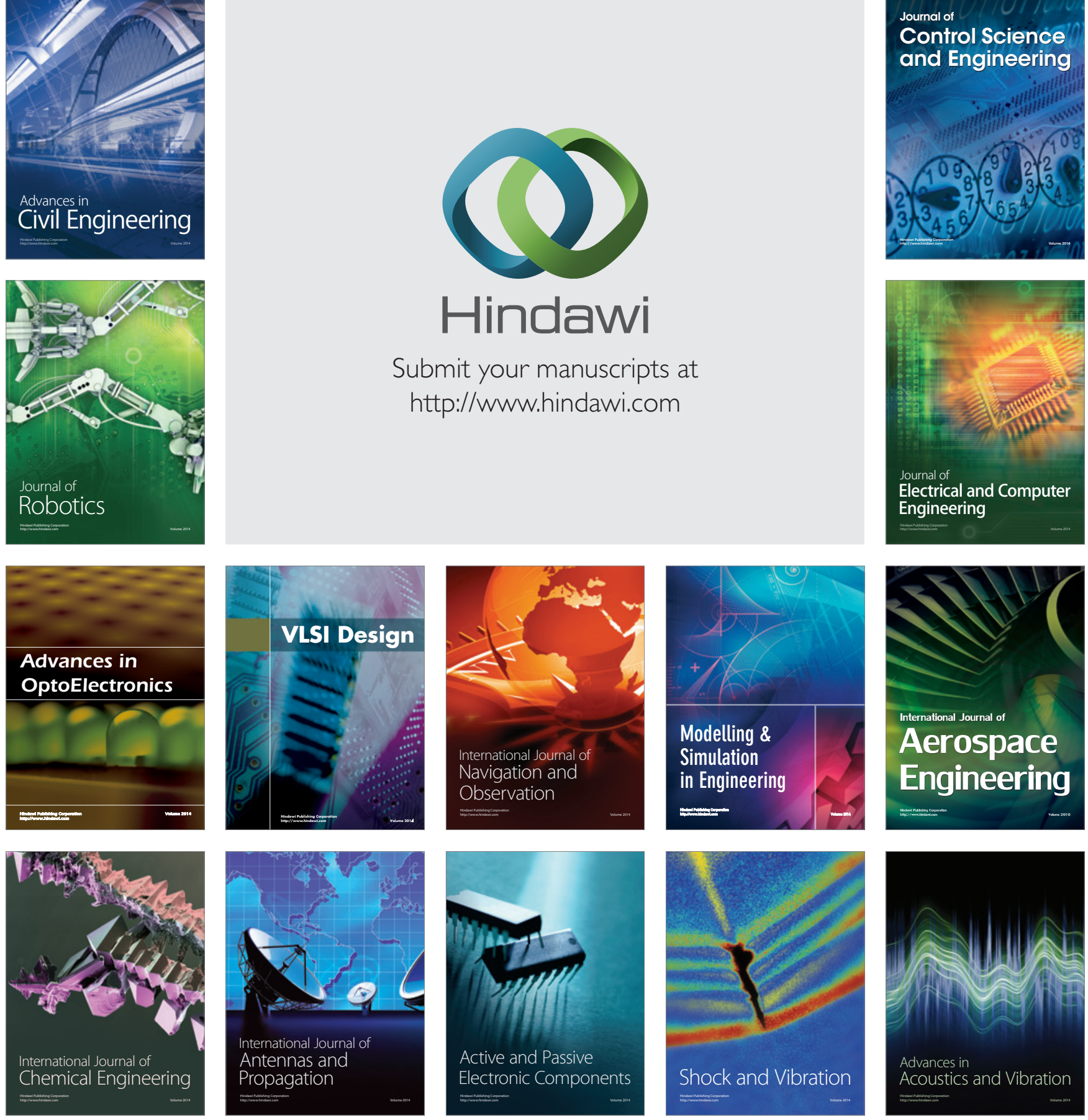